\title{
$\gamma$-Secretase inhibitor DAPT attenuates intimal hyperplasia of vein grafts by inhibition of Notch1 signaling
}

\author{
Yong Guang Xiao, Wei Wang, Dan Gong and Zhi Fu Mao
}

The proliferation and high plasticity of vascular smooth muscle cells (VSMCs) are the major reasons for restenosis of vein grafts. N-[N-(3, 5-difluorophenacetyl)-I-alanyl]-S-phenylglycine t-butyl ester (DAPT), specific inhibitor of $\gamma$-secretase, has been shown to regulate VSMC proliferation and differentiation through the Notch signaling pathway, but the pathophysiological importance of these findings in venous grafts has not yet been determined. A rat vein graft model was employed wherein the left jugular vein was surgically interposed into the left common carotid artery. Daily subcutaneous injections of DAPT or placebo (DMSO) were administered postoperatively (control animals received no treatment). We showed that DAPT can inhibit restenosis of vein grafts by inhibiting VSMC proliferation and increasing apoptosis in vivo. Notch1 signaling was highly active during the development of intima thickening. By blocking the Notch signaling pathway, the $\gamma$-secretase inhibitor DAPT can significantly attenuated intima thickening. These changes in vein grafts coincided with enhanced binding of myocardin to the smooth muscle-specific protein SM22 and smooth muscle myosin heavy chain at the promoters of VSMC differentiation-specific genes. These studies showed that DAPT can restore the VSMC phenotype and inhibit VSMC proliferation through suppression of the Notch1 signaling pathway, and thus opens a new avenue for the treatment of restenosis in vein grafts.

Laboratory Investigation (2014) 94, 654-662; doi:10.1038/labinvest.2014.58; published online 21 April 2014

KEYWORDS: $\gamma$-secretase inhibitor; intimal hyperplasia; vein graft

Veins are the most common conduits used for surgical revascularization in vascular stenosis diseases such as peripheral arterial and coronary arteriosclerosis. ${ }^{1,2}$ However, several lines of evidence have suggested that about $40 \%$ of these autologous grafts are ultimately unsuccessful, as pathologic vascular restenosis and wall thickening re-occurs, a process called intimal hyperplasia (IH). ${ }^{3-5}$ The pathogenesis of the intimal hyperplastic lesion is still poorly understood and no successful clinical intervention has been identified. It is believed that restenosis is a result of vascular smooth muscle cell (vSMC) proliferation and differentiation. ${ }^{6}$ Furthermore, these vSMCs are highly plastic and capable of modulating the transition from the contractile phenotype to pro-proliferative and antiapoptotic phenotypes in response to extracellular cues. ${ }^{7}$ However, little is known about the genetic pathways that regulate proliferation and plasticity of vSMCs in grafted veins.

The Notch signaling pathway is an evolutionarily conserved system that can regulate cell differentiation, proliferation, and survival depending on the organ and tissue. ${ }^{8,9}$ Receptor proteins of the Notch family are expressed on the cell surface and activated by five Notch ligands, including Jagged-1 and -2 and Delta-1, -2 , and $-3 .{ }^{10}$ Of the Notch receptors, only Notch1 and 3 are expressed in vasculature and are critical in regulation of vascular morphogenesis and function during development and disease. ${ }^{11}$ Furthermore, Notch1 has recently been demonstrated to have the capability to mediate vSMC proliferation and neointimal formation following vascular injury. Ligand binding to a receptor triggers a conformational change, resulting in a proteolytic cleavage and the release of the intracellular domain of Notch (NICD), which translocates into the nucleus and interacts with DNA-binding proteins. This transcriptional activator complex induces transcription of target genes, most notably the Hey and Herp genes, which regulate cell fate. ${ }^{12}$

$\mathrm{N}$-[N-(3, 5-difluorophenacetyl)-1-alanyl]-S-phenylglycine t-butyl ester (DAPT), a potent and specific inhibitor of

Department of Thoracic Surgery, Renmin Hospital of Wuhan University, Wuhan, Hubei Province, China

Correspondence: Dr YG Xiao, MD, PhD, Department of Thoracic surgery, Renmin Hospital of Wuhan University, 99 Zhangzhidong Road, Wuchang, Wuhan, Hubei Province 430060, China. E-mail:yongguangxiao@gmail.com

Received 11 November 2013; revised 14 January 2014; accepted 18 February 2014 
$\gamma$-secretase, can block the proteolytic processing and reduce the release of NICD. ${ }^{13}$ By blocking the Notch signaling pathway, DAPT can significantly inhibit vSMCs proliferation and differentiation. ${ }^{14}$ Although it has been shown that DAPT can attenuate pulmonary arterial hypertension, the pathophysiological correlate of these findings in vein graft has not been demonstrated..$^{15}$ In the present study, we proposed that Notch signaling is crucial during the process of IH in vein grafts. By establishing a rat model of vein graft by interposing the left jugular vein into the left common carotid artery, we investigated the role of DAPT in the development of IH by regulating vSMCs proliferation and differentiation.

\section{MATERIALS AND METHODS Animal Studies}

Male wistar rats (250-350 g) were purchased from Wuhan Animal Center and fed with standard rat chow and tap water ad libitum. All animal protocols were approved by the Animal Care and Use Committee of Renmin Hospital of Wuhan University.

Under the microscope, these rats were interposed the left jugular vein into the left common carotid artery. Briefly, after anesthesia by intraperitoneal administration of $30 \mathrm{mg}$ per $\mathrm{kg}$ body weight sodium pentobarbital, all rats were given a single intravenous dose of heparin $(1.5 \mathrm{mg} / \mathrm{kg})$. Next, the left carotid artery and jugular vein were dissected via a vertical mid-line, and a $1-\mathrm{cm}$ segment of jugular vein was harvested and flushed with saline solution $\left(4^{\circ} \mathrm{C}\right)$ containing heparin $(5 \mu / \mathrm{ml})$. Then the carotid artery was cut after occlusion on both sides by microvascular clips. Finally, the jugular vein graft was anastomosed into the carotid artery, interposed in a reverse end-to-end fashion, using 11-0 polypropylene sutures. Rats either received no further treatment (control), or a daily subcutaneous injection of DAPT $(0,5$, and $10 \mathrm{mg} /$ $\mathrm{kg}$ ) or placebo (DMSO, $10 \mathrm{mg} / \mathrm{kg}$ ) from day 0 to day 28 postsurgery. Although gastrointestinal side effects have been reported for $\gamma$-secretase inhibitor administration in mice, we did not observe any overt side effects of DAPT administration at this dose. ${ }^{16}$

\section{Histological and Immunofluorescence Analyses}

These rats were anaesthetized by the method as described earlier, and the venous grafts of rats were harvested at the given time point. Grafts were obtained by cutting the transplanted segments from the native vessels at the cuff end. For histological analysis, vein grafts were perfusion-fixed. Then, samples were fixed with $4 \%$ phosphate-buffered formaldehyde at $4{ }^{\circ} \mathrm{C}$ for $24 \mathrm{~h}$ and embedded in paraffin. For vascular morphometry, paraffin-embedded vein graft blocks were cut into $4 \mu \mathrm{m}$ sections and stained with hematoxylin and eosin. Intima thickness area of vein grafts was measured with a microscope digital camera system (Zeiss). Intima area was calculated by the following formula: intima area $=$ areas bounded by internal elastic lamina-lumen. Media area was calculated by the following formula: media area $=$ areas bounded by external elastic lamina-areas bounded by internal elastic lamina. The intima/media ratio was calculated by the following formula: $\mathrm{I} / \mathrm{M}$ ratio $(\%)=$ intima area/media area $\times 100$.

For vein graft staining of proliferating cell nuclear antigen (PCNA), the $4 \mu \mathrm{m}$ sections were permeabilized with $0.1 \%$ PBS for $30 \mathrm{~min}\left(4^{\circ} \mathrm{C}\right)$, and then incubated with anti-PCNA antibody (Abcam, 1:600) for $1 \mathrm{~h}$ at room temperature, followed by incubation with an Alexa Flour labeled secondary antibody at a 1:300 dilution. Quantitative analysis of PCNApositive cells was performed by counting the positive nuclei in vSMCs from five randomly selected fields of the grafted venous intima under a microscope digital camera system at $\times 200$. The average of the five determinants was calculated and was recorded as the number of PCNA-positive cells per square micrometer. The results were expressed as the proliferation index and calculated by the following formula: the percentage of PCNA-positive nuclei $=($ total PCNA-positive cells/total cell count) $\times 100 \%$.

For vein graft terminal deoxynucleotidyl transferase dUTP nick-end labeling (TUNEL) staining, pretreated sections were incubated with the TUNEL reaction mixture (Roche, Germany) for $1 \mathrm{~h}$ at room temperature, next followed by incubation with DAB. Apoptotic cells that appeared as green were visualized with a microscope. Quantification of TUNEL-positive cells was performed in the same manner as PCNA-positive cells.

\section{Cell Culture}

Rat vSMCs were cultivated from venous grafts using collagenase and elastase as described previously. ${ }^{17}$ In short, venous grafts were harvested using sterile technique and washed with RPMI 1640 medium. The intima and inner twothirds of the media were carefully dissected from the venous graft under the microscope, cut into pieces, and then were digested in DMEM containing collagenase subtype $(1 \mathrm{mg} / \mathrm{ml}$, Roche, USA) for $4 \mathrm{~h}$ at $37^{\circ} \mathrm{C}$. The resulting solution was centrifuged, and the pellet was resuspended in DMEM containing $20 \% \mathrm{FBS}$ and then seeded onto $100-\mathrm{mm}$ culture dishes. The dishes were incubated upside down at $37^{\circ} \mathrm{C}$ in a humidified atmosphere of $95 \%$ air $/ 5 \% \mathrm{CO}_{2}$.

Rat vSMCs were dissociated with 1-mg/ml collagenase into single cells and cultured in DMEM containing 0.1\% DMSO or $5 \mu \mathrm{M}$ DAPT for 7 days at a density of $1 \times 10^{5} \mathrm{cells} / \mathrm{ml}$. Fifty percent of the medium was replaced every $2 \sim 3$ days. ${ }^{18}$

\section{Quantitative Real-Time PCR, ChIP-PCR, and Western Blot} Quantitative real-time PCR was used for quantitative analysis of Notch1 ICD mRNA level in vSMCs infected with Notch1 ICD adenovirus or lacZ adenovirus. In brief, total cellular RNA was extracted using RNeasy Mini Kit (Qiangen Sciences) and reversed-transcribed into cDNA and amplified using TaqMan Reverse Transcription reagents (Applied Biosystems). Quantitative analysis of gene expression was 
performed using the ABI Prism 7700 Sequence Detection System with $18 \mathrm{~s}$ rRNA used as an endogenous reference.

The chromatin immunoprecipitation (ChIP) assay was performed according to the Abcam X-ChIP protocol. In brief, cross-linked chromatin from subcultured vSMCs was sonicated to generate 500-1000 bp DNA fragments, followed by overnight incubation at $4{ }^{\circ} \mathrm{C}$ with primary antibodies specific for myocardin (Abcam) or IgG (Santa Cruz, negative control). Immunoprecipitated DNA was purified and amplified by RT-PCR with primers flanking the CArG boxes of smooth muscle $22 \alpha$ (SM 22 $\alpha$ ) and smooth muscle myosin heavy chain (SMMHC). Primer sequences were as follows: SM22: $5^{\prime}$-GGTCCTGCCCATAAAAGGTTT- ${ }^{\prime}$ and $5^{\prime}$-TGCCCATGG AAGTCTGCTTGG-3'; SMMHC: 5'-CTGCGCGGGACCATA TTTAGTCAGGGGGAGG- $3^{\prime}$ and $5^{\prime}$-CTGGGCGGGAGACAA CCCAAAAAGGCCAGG-3'

Protein levels of Bcl-2, Cyclin D1, Nocth3 ICD, HES-5 and SM22, SMMHC, syndcan-1, myocardin in cell lysate were evaluated by an ECL Western blotting analysis kit (Amersham Biosciences). The primary antibodies used in this study were against: $\beta$-actin (Santa Cruz, 1:2000), Bcl-2 (Abcam, 1:400), Cyclin D1 (Abcam, 1:400), Nocth3 ICD and HES-5 (Santa Cruz, 1:1000), SM22 $\alpha$ and SMMHC (Abcam, 1:800), Syndecan-1 (Santa Cruz, 1:600), Vimentin (Abcam, 1:600), myocardin (Abcam, 1:600).

\section{Adenovirus Vector Production and Transduction}

cDNA containing the entire full-length Notch1 ICD or LacZ was used to construct and produce recombinant AdNEP plasmid (AdEasy Adenoviral Vector System, Stratagene). Adenovirus vectors were amplified and purified by cesium chloride density gradient ultracentrifugation. First, vSMCs were seeded at well for $12 \mathrm{~h}$, and then washed three times with PBS before the addition of SMGM without fetal bovine serum. Cells were incubated at $37{ }^{\circ} \mathrm{C}$, with $5 \% \mathrm{CO}_{2}$ for $6 \mathrm{~h}$ and then treated with adenoviruses. The AdLacZ vector was used as a negative control.

\section{Statistical Analysis}

Data are expressed as Mean \pm s.e.m., and statistical analyses were performed with the Prism statistical program. Unpaired Student's- $t$ test was used for comparisons between two means. One-way analysis of variance with the NewmanKeul's was used to evaluate differences between more than two means. In all case, $P$-value less than 0.05 was considered significant. All the histological measurements in regard to vascular morphometry were performed by two observers in a blinded manner, and the values they obtained were averaged.

\section{RESULTS}

$\gamma$-Secretase Inhibitor DAPT Attenuates IH of Vein Grafts

First we investigated the effect of DAPT on IH in the rat model of vein grafts. At days 14 and 28 after the model was established, the interposing venous graft was harvested and subjected to hematoxylin and eosin staining. All veins manifested progressively worse IH over a 28-day period following grafting (Figure 1a). We found no significant difference in the $\mathrm{IH}$ of grafted veins among the different group on day 14 post-surgery. But at day 28 post-surgery, the administration of DAPT had obviously ameliorated the intimal thickness in a dose-dependent manner (Figure 1b). Consistent with this observation, DAPT can also serve as a protective mechanism against increased intima/media (I/M) ratio (Figure 1c) and decreased intima area (Figure 1d). In addition, our studies showed that the morphology was not altered in both normal vein and artery by the administration of DAPT. Combined, these findings indicate that $\gamma$-secretase inhibitor DAPT treatment served a protective role against IH from the initial stage of the vein graft model.

Next, we tested the therapeutic potential of DAPT in reversal of IH. A daily subcutaneous injection of $10 \mathrm{mg}$ per $\mathrm{kg}$ body weight DAPT or DMSO (placebo) was given to rats from day 14 post-surgery when IH had already been well established in the vein graft model. We found that the daily administration of DAPT did not completely reverse, but did exhibit a strong therapeutic effect in the rat model of vein graft as compared with the DMSO administration. Treatment halted progression of $\mathrm{IH}$ as judged by measurement of intimal thickness (Figure 1e), intimal/media ratio (Figure 1f) and intima area (Figure 1g). Taken together, our studies confirm that $\gamma$-secretase inhibitor DAPT administration can protect against formation and development of IH in the vein graft model.

\section{DAPT Regulates vSMCs Proliferation and Apoptosis}

As enhanced proliferation of vSMCs is the most important underlying mechanisms in restenosis of vein grafts, we investigated whether DAPT treatment can inhibit IH by regulating vSMCs fate through the Notch signaling pathway in vein grafts in vivo and in vitro. Using intracellular staining with PCNA and TUNEL, we evaluated the effect of DAPT on vSMCs proliferation and apoptosis of vein grafts in vivo. As shown in Figure 2a, expression of PCNA was significantly decreased in DAPT-treated vein grafts as compared with control and DMSO-treated vein grafts. In contrast, expression of TUNEL was excessively increased in DAPT-treated vein grafts as compared with control and DMSO-treated vein grafts (Figure 2b). These data directly reflect proliferative and apoptotic rates for vSMCs of grafted vein in vivo. To further confirm that DAPT can inhibit vSMCs proliferation in vein grafts in vivo, the cell cycle and proliferative capacity were measured. We compared protein and mRNA levels of both Bcl-2 and cyclin D1 in the vSMCs among control, DAPT- and DMSO-treatment vein graft. Our results show that DAPT clearly reduces mRNA expression of both Bcl-2 and cyclin D1 as compared with the control and DMSO-treated vein grafts (Figure 2c). The RT-PCR data were consistent with western blotting results, revealing that both the control and DMSOtreated vein graft exhibited strong Bcl-2 and cyclin D1 

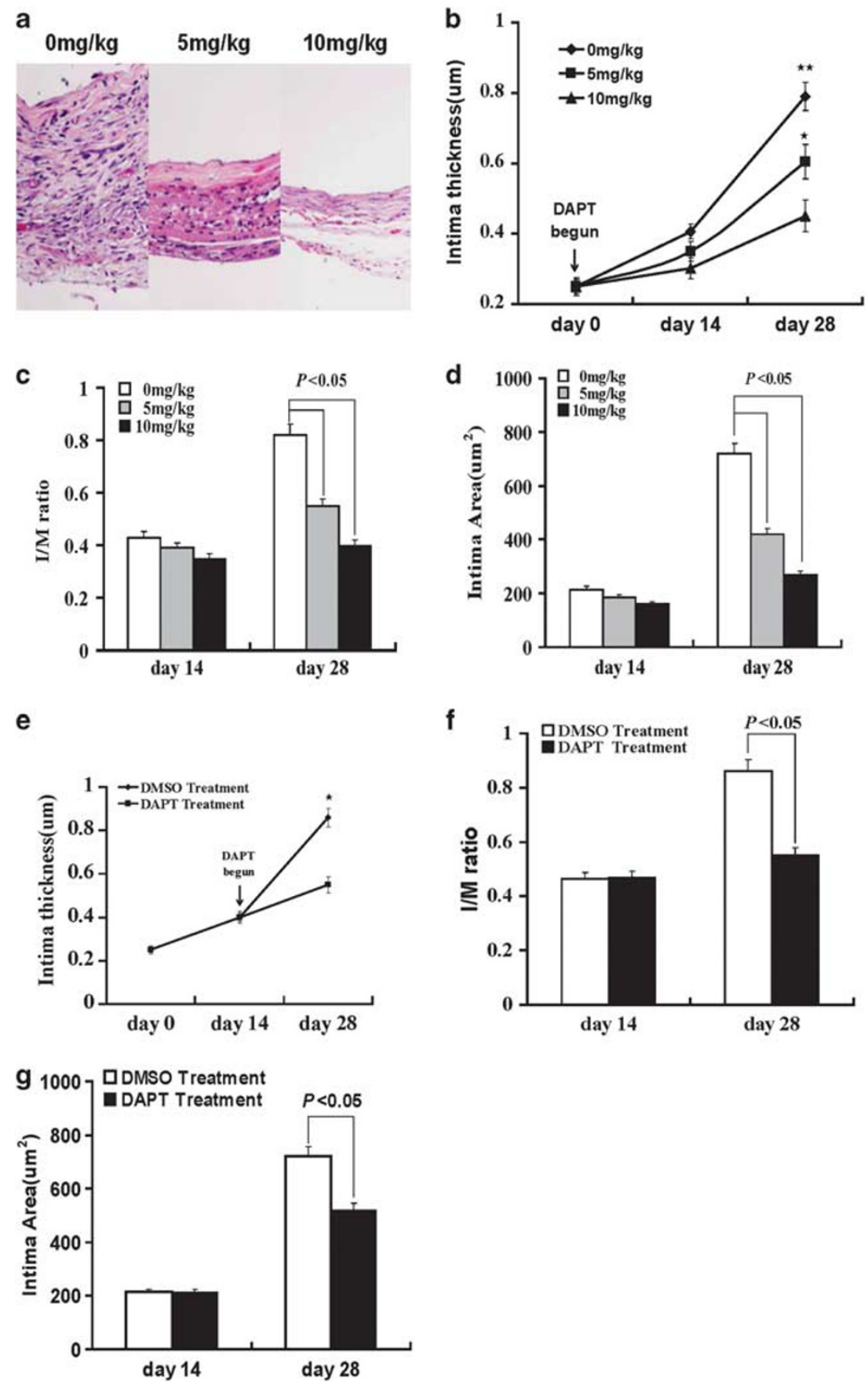

Figure 1 Rat vein graft models were established and $\mathrm{N}$-[N-(3, 5-difluorophenacetyl)-l-alanyl]-S-phenylglycine t-butyl ester (DAPT) was then administered as a daily subcutaneous dose of 0,5 , and $10 \mathrm{mg} / \mathrm{kg}$ from day 0 to day 28 post-surgery. In (a) representative hematoxylin and eosin (H\&E)-stained sections of a vein graft from rat on day 28 (scale bars; $30 \mu \mathrm{m}$ ). Intima thickness (b), intima/media (I/M) ratio (c), and intima area (d) of vein grafts in rats were measured at various time points. In separate studies, DAPT was given to animals in the same way on day 14 post-surgery and the level of intima thickness $(\mathbf{e}), \mathrm{l} / \mathrm{M}$ ratio $(\mathbf{f})$, and intima area $(\mathbf{g})$ of vein grafts were measured at indicated time points. Data are means \pm s.e.m.; $n=8$ rats or independent experiments per time point for each group, 10 sections per rat. In $\mathbf{b},{ }^{*} P<0.055 \mathrm{vs} 0 \mathrm{mg} / \mathrm{kg},{ }^{* *} P<0.0110 \mathrm{vs} 0 \mathrm{mg} / \mathrm{kg}$. In e, ${ }^{*} P<0.05 \mathrm{DMSO}$ treatment vs DAPT treatment.

protein expression (Figure $2 \mathrm{~d}$ ). The increased protein expression indirectly reflected the proliferative capacity of vSMCs in vein grafts. Direct evidence for the inhibitory activity of
DAPT on vSMCs proliferative capacity was obtained by cell culture experiments. As shown in Figure 2e, DAPT treatment can halt subcultured vSMCs to roughly half the proliferation 

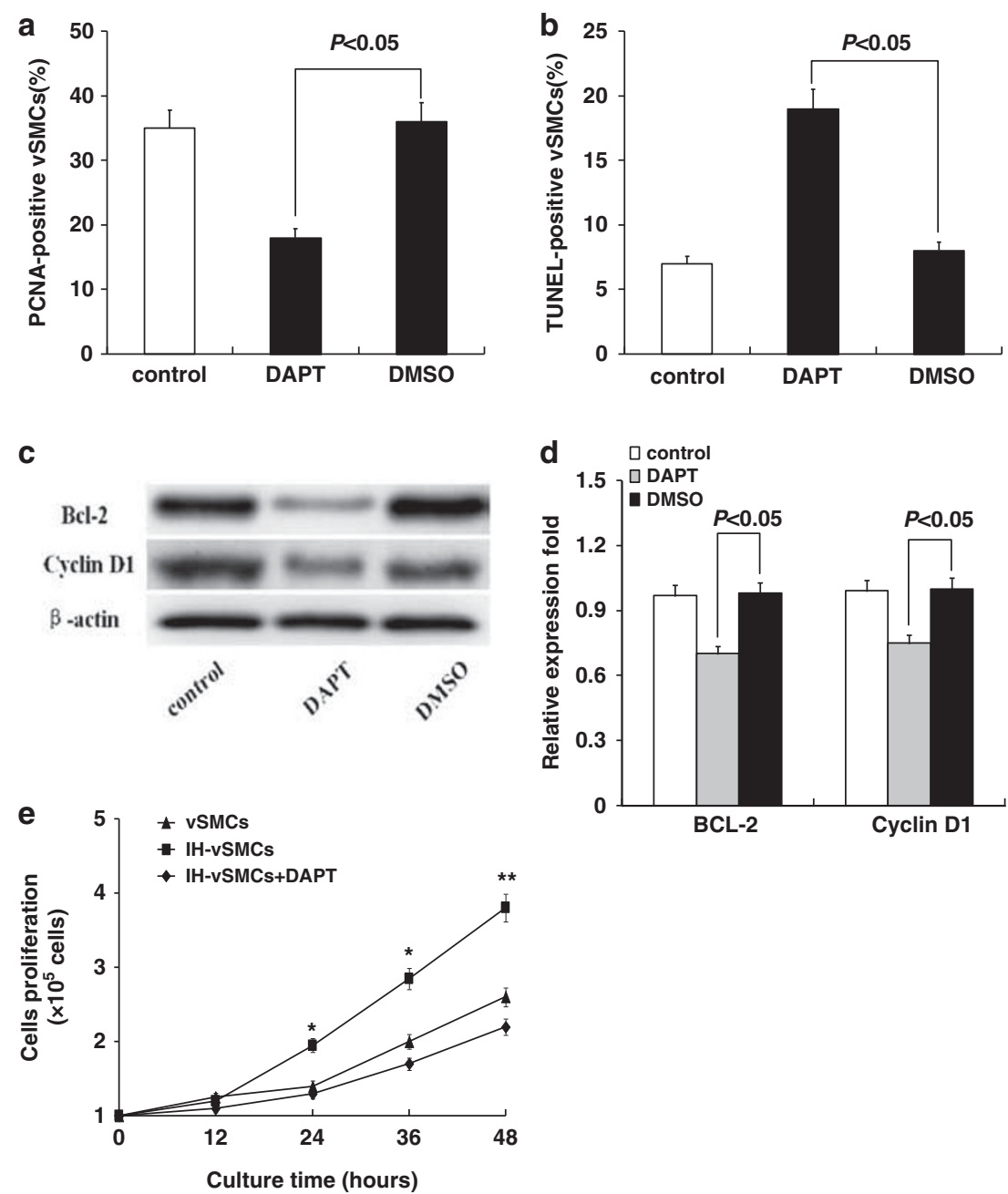

Figure 2 Rat vein graft models were established, and daily subcutaneous doses of $10 \mathrm{mg} / \mathrm{kg} \mathrm{N}$-[N-(3, 5-difluorophenacetyl)-l-alanyl]-S-phenylglycine t-butyl ester (DAPT) or dimethlysulphoxide (DMSO) were administered from day 0 to day 28 post-surgery. Control animals received no post-surgical treatment. The percentage of vascular smooth muscle cells (vSMCs) that are proliferating cell nuclear antigen (PCNA)-positive (a) and terminal deoxynucleotidyl transferase dUTP nick-end labeling (TUNEL)-positive (b) in all types of veins was obtained by immunohistochemical analysis. In c, western blot analysis shows the protein levels of Bcl-2 and Cyclin D1 in three vein grafts. In d, relative expression values obtained by densitometry of $\mathrm{Bcl}-2$ and Cyclin D1 protein are normalized to $\beta$-actin (bottom).The vSMCs from normal veins, vein grafts, and DAPT-treated vein grafts were subcultured for determination of growth curves (e). Data are means \pm s.e.m.; $n=8$ rats. In $\mathbf{e},{ }^{*} P<0.05,{ }^{*} P<0.01$ vs vSMCs and intimal hyperplasia (IH)-vSMCs + DAPT.

rate as compared with those without treatment. Combined, these results indicate that the therapeutic effect of DAPT on $\mathrm{IH}$ involves both anti-proliferative and pro-apoptotic effects on vSMCs of vein grafts.

\section{Proliferation of vSMCs is Dependent on Notch1 Signaling}

Next, we examined expression of the Notch family of proteins in vein grafts. Western blot data showed that it was Notch1 protein, not Notch3, which increased gradient expression in vSMCs of vein grafts (Figure $3 a$ and $b$ ). As the $\gamma$-secretase inhibitor DAPT had been shown to block Notch signaling by inhibiting the cleavage of Notch protein to intracellular domain (ICD) peptides both in vitro and in vivo, we tested whether the effect of DAPT on IH of vein graft was dependent on Notch1 signaling. Western blots revealed that DAPT inhibited the expression of Notch1 ICD (NICD) protein in a dose-dependent manner (Figure $3 \mathrm{c}$ and $\mathrm{d}$ ). Then, we investigated whether there was a direct involvement of Notch1 ICD in the development of proliferative vSMCs. Subcultured wild-type (WT) vSMCs infected with the Notch1 ICD adenovirus (amino-acid sequence for NICD) showed increased Notch1 ICD mRNA expression as compared with lacZ-transduced WT vSMCs (Figure $3 e$ and f). 

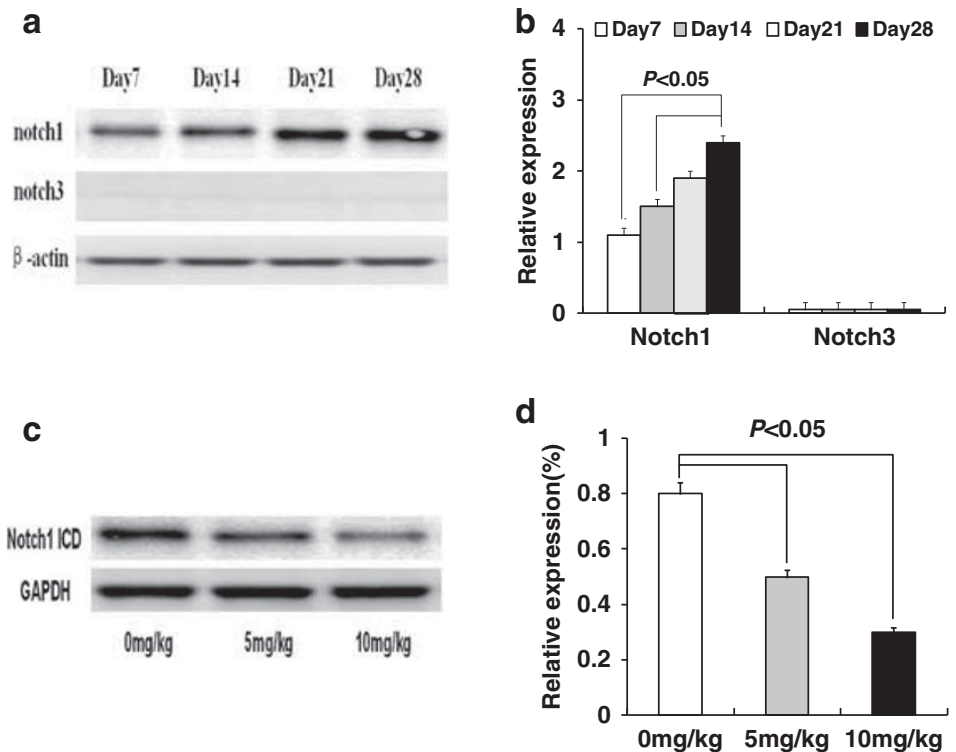

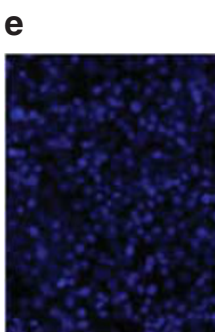

Adeno-lac Z

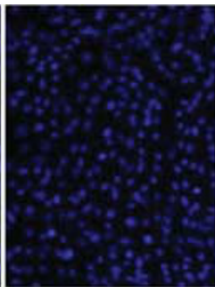

Adeno-NICD

\section{g}

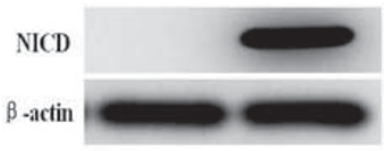

Adeno-lacZ Adeno-NICD

$\mathbf{f}$

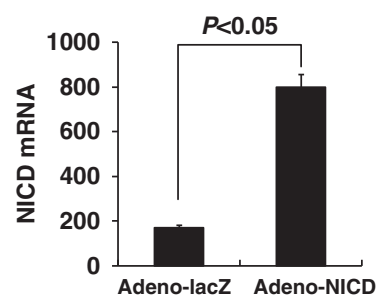

h

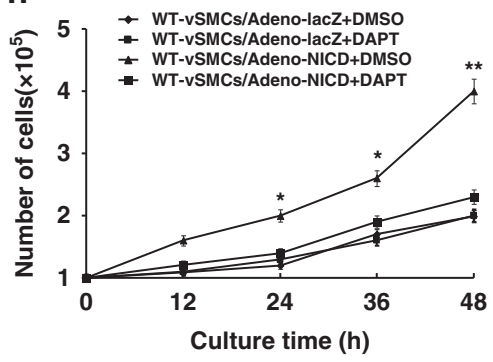

Figure 3 (a) Western blot analysis shows the protein levels of Notch1 and Notch3 in vascular smooth muscle cells (vSMCs) at indicated time points after establishment of vein grafts. In $\mathbf{b}$, relative expression values obtained by densitometry of notch 1 and notch 3 protein were normalized to $\beta$-actin (bottom). In c, rat vein grafts were harvested on day 28 after daily subcutaneous administration N-[N-(3, 5-difluorophenacetyl)-I-alanyl]-S-phenylglycine t-butyl ester (DAPT) at doses of 0,5 , and $10 \mathrm{mg}$ per kg body weight from day 0 to day 28 post-surgery; western blot analysis showed Notch1 intracellular domain (ICD) expression in all vein grafts. (d) Relative expression values were obtained by densitometry of Notch1 ICD protein normalized to glyceraldehyde 3-phosphate dehydrogenase (GAPDH) (bottom). In e, immunohistochemical analysis of intracellular domain of Notch (NICD) expression in VSMC subculture infected with adenovirus containing the CDNA for NICD (right) compared with the same subculture infected with lacZ adenovirus (left). The vSMCs subculture infected with NICD and lacZ adenoviruses were analyzed for NICD expression by RT-PCR (f) and western blot (g). In $\mathbf{h}$, growth curves of vSMCs-infected NICD adenoviruses or lacZ adenoviruses, treated with DAPT (20 $\mu$ mol/l) or DMSO (20 $\mu \mathrm{mol} / \mathrm{l})$. Data are means \pm s.e.m.; ${ }^{*} P<0.05,{ }^{*} P<0.01$ vs wild-type (WT) vSMCs/Adeno-lacZ + DMSO, WT vSMCs/Adeno-lacZ + DAPT and WT vSMCs/Adeno-NICD + DAPT.

The RT-PCR data were reflected by western blotting, revealing that WT vSMCs infected with the Notch1 ICD adenovirus exhibited strong Notch1 ICD protein expression (Figure 3g). Analysis of the effects of constitutive, high-level
Notch1 ICD expression in vSMCs revealed a significantly increased growth rate (Figure $3 \mathrm{~h}$ ). These results suggest that upregulated notch1 signaling has a major role in the proliferation of vSMCs from vein graft. 


\section{DAPT Restored Phenotypic Pattern of vSMCs in Vein Graft}

To gain a better understanding of the molecular pathways involved in DAPT inhibition of IH-vSMCs proliferation, we compared phenotypic patterns of vSMCs among normal vein and vein grafts without and with DAPT treatment. In vSMCs of vein grafts, expression levels of proteins associated with differentiated/contractile phenotype including smooth muscle $22 \alpha$ (SM 22 $\alpha$ ) and smooth muscle myosin heavy chain (SMMHC) were decreased in comparison to the vSMCs of normal vein and DAPT-treated vein graft (Figure $4 \mathrm{a}$ and $\mathrm{b}$ ). In contrast, the vSMCs dedifferentiated/pro-proliferative/ synthetic-specific marker proteins including syndecan- 1 and vimentin were more abundant in vSMCs of vein graft relative to normal vein and DAPT-treated vein graft (Figure 4a and $b$ ). These changes in phenotype-specific marker proteins presented in vein graft were almost completely reversed by DAPT treatment. Taken together, these approaches provide evidence that acquisition of the proliferative characteristic by
vSMCs of vein graft during development of IH can be reversed by DAPT administration.

The mechanism by which DAPT can restore vSMCs differentiation was then examined. We evaluated myocardin protein expression by western blotting and demonstrated that DAPT can upregulate myocardin in vSMCs of vein grafts (Figure $4 \mathrm{c}$ and d). Given the effect of DAPT on myocardin, we then tested whether physical binding of myocardin to the CArG-containing regions of vSMCs-specific promoters was indeed affected by DAPT. Using quantitative ChIP assays, we found a lower level of myocardin binding to the CArGcontaining regions of the smooth muscle-specific protein SM22 and smooth muscle myosin heavy chain (SMMHC) promoters in vSMCs of vein graft as compared with normal vein and DAPT-treated vein graft. The observed effects of DAPT on vSMCs-specific transcription may explain DAPT-dependent reverse modulation of vein graft vSMCs phenotype from the dedifferentiated to the differentiated state. a

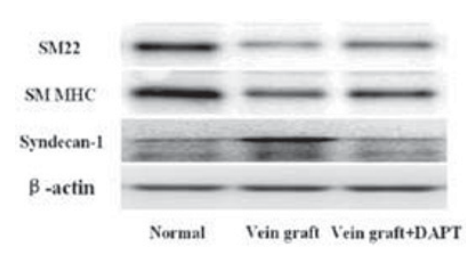

C

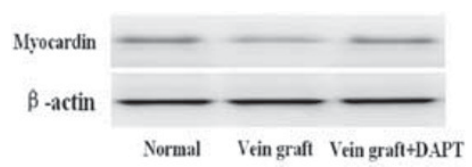

e

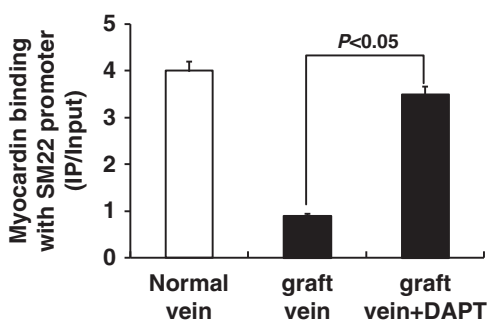

b
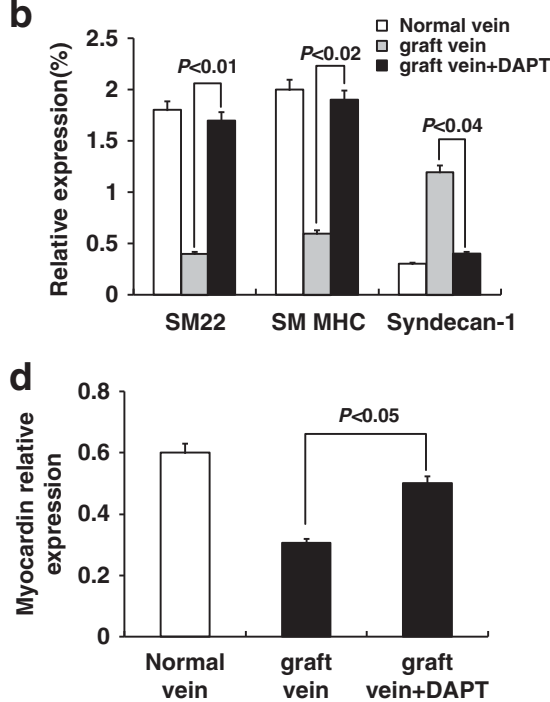

f

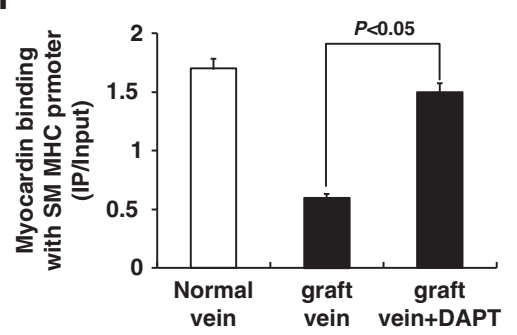

Figure 4 In a, western blot analysis of protein of smooth muscle 22 (SM22), smooth muscle myosin heavy chain (SMMHC), and syndecan-1 of vascular smooth muscle cells (vSMCs) in normal veins, vein grafts, and $\mathrm{N}$-[N-(3, 5-difluorophenacetyl)-l-alanyl]-S-phenylglycine t-butyl ester (DAPT)-treated vein graft. (b) Relative expression values obtained by densitometry of SM22, SMMHC, and syndecan-1 protein were normalized to $\beta$-actin (bottom). In c, western blot analysis of myocardin protein in normal vein, a vein graft, and a DAPT-treated vein graft. (d) Relative expression values obtained by densitometry of myocardin protein normalized to $\beta$-actin (bottom). In separate studies, these cells were processed for chromatin immunoprecipitation (ChIP) assays and RT-PCR was performed on immunoprecipitation products using primers for the CArG-containing regions of SM22- (e) and SMMHC-(f) specific promoters. Data are means \pm s.e.m. 


\section{DISCUSSION}

Our study clearly demonstrates that Notch signaling is highly activated in vein grafts and promotes development of $\mathrm{IH}$ and proliferation of vSMCs. By blocking Notch signaling, the $\gamma$-secretase inhibitor DAPT can significantly attenuate intimal thickening, while also regulating proliferation of vSMCs. We have defined the mechanisms of DAPT suppression on $\mathrm{IH}$ and vSMCs proliferation by repressing the activity of Notch1 pathway and stimulating the myocardin pathway, thereby increasing vSMCs differentiation marker expression. To our knowledge, this is the first demonstration that Notch signaling is activated to promote $\mathrm{IH}$ in experimental vein grafts model. Importantly, we provide intriguing evidence that the pathologically anti-apoptotic and proliferative vSMCs phenotype in vein graft can be reversed toward the normal vSMCs by administration of DAPT.

Veins are considered the best choice for vascular graft conduits, particularly in the setting of arterial reconstructive surgery for patients with coronary artery disease and peripheral arterial ischemia. ${ }^{19}$ The vessel wall thickening of the vein graft is the adaptive response to the arterial environment during the post-surgical process with deposition of excessively proliferative vSMCs and extracellular matrix in all layers of the vein graft, especially in the intima. ${ }^{20}$ However, arteries and veins are anatomically, functionally, and molecularly distinct, and the vein is specifically characterized by the suppression of Notch signaling. ${ }^{21}$ This theory is supported by our results that show that vascular remodeling after vein grafting correlates with the activity of the Notch pathway. It is worth while to point out that DAPT has been shown to inhibit the proliferation of vSMCs by reducing both Bcl-2 and cyclin D1 expression depending on notch1 signaling. This finding may be significant as a previous study had demonstrated that cyclic strain inhibits vSMCs growth, while enhancing vSMCs apoptosis through regulation of Notch receptor and downstream target gene expression. ${ }^{22,23}$ Our study demonstrated that the administration of DAPT may also induce apoptosis in the vSMCs, thereby worsening IH. This result is accordant with previous reports that silencing Notch1 can induce cell apoptosis through Bcl-2. ${ }^{24,25}$ Collectively, the intimal thickening protective effects provided by DAPT are likely through multiple pathways, which regulate vSMCs proliferation and apoptosis. Some of these pathways do not overlap, and this feature is important because it may reduce the likelihood of emergence of therapy-resistance. In support of this notion, studies from other groups have shown that therapeutic interventions targeting either vSMCs apoptosis $^{26,27}$ or proliferation ${ }^{28,29}$ could regress experimental intima thickening.

Our results also reveal that DAPT can restore vSMC transition from a dedifferentiated phenotype to a differentiated/contractile phenotype by enhancing binding of myocardin to a marker protein, such as SM22 and SMMHC. Unlike skeletal and cardiac muscle cell lineages, where cellular differentiation is functionally coupled to irreversible exit from the cell cycle, vSMCs retain their capacity to proliferate and modulate their phenotype in response to extracellular cues. $^{30}$ Previous reports have demonstrated that Notch signaling in vSMCs is important during vascular repair of injury and vascular pathology. ${ }^{31}$ vSMCs express multiple Notch receptors throughout their life cycle, and respond to Notch ligands as a regulatory mechanism of differentiation and maturation. ${ }^{8,32}$ DAPT, as $\gamma$-secretase inhibitor, has been shown to block the cleavage of Notch protein to ICD, inhibit the capable of reversible change in vSMCs phenotype from a dedifferentiated/proliferative state to differentiated/contractile state as seen in normal vSMCs.

Our study demonstrates that TUNEL activity is increased in DAPT-treated vein grafts. This shows that DAPT can induce apoptosis of vSMCs in IH. This is not an isolated finding; previous studies have clearly demonstrated that Notch signal suppresses apoptosis in pancreatic islet and tumor cells. ${ }^{33,34}$ The $\gamma$-secretase inhibitor DAPT can inhibit Notch signaling by reducing the expression of NICD protein. These findings are in accordance with enhancement of the apoptosis activity in DAPT-treated vein grafts.

DAPT, as a potent and specific inhibitor of $\gamma$-secretase inhibitor, has safety drawbacks. The $\gamma$-secretase inhibitor can result in an early embryonal lethality with the phenotype presenting skeletal and central nervous system defects similar to those of a Notch knockout. It is known that Notch signaling has an important role in the ongoing differentiation processes of the immune system and of the gastrointestinal tract. ${ }^{35,36}$ But we did not observe any overt side effects with DAPT administration at our study.

In summary, our work provides a specific therapeutic strategy for attenuation and reversal of multiple pathological processes that are associated with intima thickening of vein grafts, through administration of $\gamma$-secretase inhibitor DAPT to inhibit Notch signaling.

\section{DISCLOSURE/CONFLICT OF INTEREST}

The authors declare no conflict of interest.

1. Hillis LD, Smith PK, Anderson JL, et al. 2011 ACCF/AHA Guideline for Coronary Artery Bypass Graft Surgery: executive summary: a report of the American College of Cardiology Foundation/American Heart Association Task Force on Practice Guidelines. Circulation 2011;124: 2610-2642.

2. Serruys PW, Morice MC, Kappetein AP, et al. SYNTAX Investigators. Percutaneous coronary intervention versus coronary-artery bypass grafting for severe coronary artery disease. N Engl J Med 2009;360: 961-972.

3. Serruys PW, Unger F, Sousa JE, et al. Arterial Revascularization Therapies Study Group. Comparison of coronary-artery bypass surgery and stenting for the treatment of multivessel disease. N Engl J Med 2001;344:1117-1124.

4. Harskamp RE, Lopes RD, Baisden $C E$, et al. Saphenous vein graft failure after coronary artery bypass surgery: pathophysiology, management, and future directions. Ann Surg 2013;257:824-833.

5. Kudo FA, Muto A, Maloney SP, et al. Venous identity is lost but arterial identity is not gained during vein graft adaptation. Arterioscler Thromb Vasc Biol 2007;27:1562-1571. 
6. Bikdeli B, Hassantash SA, Pourabdollah M, et al. Histopathologic insight into saphenous vein bypass graft disease. Cardiology 2012;123. 208-215.

7. Owens GK, Kumar MS, Wamhoff BR. Molecular regulation of vascular smooth muscle cell differentiation in development and disease. Physiol Rev 2004;84:767-801.

8. Morrow D, Guha S, Sweeney C, et al. Notch and vascular smooth muscle cell phenotype. Circ Res 2008;103:1370-1382.

9. Boucher J, Gridley T, Liaw L. Molecular pathways of notch signaling in vascular smooth muscle cells. Front Physiol 2012;3:81.

10. Roca C, Adams RH. Regulation of vascular morphogenesis by Notch signaling. Genes Dev 2007;21:2511-2524.

11. Chen Y, Zheng S, Qi D, et al. Inhibition of Notch Signaling by a c-Secretase Inhibitor Attenuates Hepatic Fibrosis in Rats. PLOS ONE 2012;7:e46512.

12. Proweller A, Pear WS, Parmacek MS. Notch signaling represses myocardin-induced smooth muscle cell differentiation. J Biol Chem 2005;280:8994-9004.

13. Li Y, Takeshita K, Liu PY, et al. Smooth muscle Notch1 mediates neointimal formation after vascular injury. Circulation 2009;119: 2686-2692.

14. Liu H, Kennard S, Lilly B. NOTCH3 expression is induced in mural cells through an autoregulatory loop that requires endothelial-expressed JAGGED1. Circ Res 2009;104:466-475.

15. Li X, Zhang $X$, Leathers $R$, et al. Notch3 signaling promotes the development of pulmonary arterial hypertension. Nat Med 2009;15: 1289-1297.

16. Real PJ, Tosello V, Palomero $\mathrm{T}$, et al. Gamma-secretase inhibitors reverse glucocorticoid resistance in T-ALL. Nat Med 2009;15: 50-58.

17. Sun J, Zheng J, Ling $\mathrm{KH}$, et al. Preventing intimal thickening of vein grafts in vein artery bypass using STAT-3 siRNA. J Transl Med 2012;10:2.

18. Woo SM, Kim J, Han HW, et al. Notch signaling is required for maintaining stem-cell features of neuroprogenitor cells derived from human embryonic stem cells. BMC Neurosci 2009;10:97.

19. Sabik 3rd JF. Understanding saphenous vein graft patency. Circulation 2011;124:273-275.

20. Muto A, Model L, Ziegler K, et al. Mechanisms of vein graft adaptation to the arterial circulation: insights into the neointimal algorithm and management strategies. Circ J 2010;74:1501-1512.

21. You LR, Lin FJ, Lee CT, et al. Suppression of Notch signalling by the COUP-TFIl transcription factor regulates vein identity. Nature 2005 435:98-104.
22. Morrow D, Sweeney C, Birney YA, et al. Cyclic strain inhibits Notch receptor signaling in vascular smooth muscle cells in vitro. Circ Res 2005;96:567-575.

23. Campos $\mathrm{AH}$, Wang $\mathrm{W}$, Pollman $\mathrm{MJ}$, et al. Determinants of Notch-3 receptor expression and signaling in vascular smooth muscle cells: implications in cell-cycle regulation. Circ Res 2002;91:999-1006.

24. Ye QF, Zhang YC, Peng XQ, et al. Silencing Notch-1 induces apoptosis and increases the chemosensitivity of prostate cancer cells to docetaxel through Bcl-2 and Bax. Oncol Lett 2012;3:879-884.

25. Wang Z, Zhang Y, Li Y, et al. Down-regulation of Notch-1 contributes to cell growth inhibition and apoptosis in pancreatic cancer cells. Mol Cancer Ther 2006;5:483-493.

26. Zheng $\mathrm{H}$, Xue $\mathrm{S}$, Lian $\mathrm{F}$, et al. A novel promising therapy for vein graft restenosis: overexpressed Nogo-B induces vascular smooth muscle cell apoptosis by activation of the JNK/p38 MAPK signaling pathway. Med Hypotheses 2011;77:278-281.

27. Lyon CA, Koutsouki E, Aguilera CM, et al. Inhibition of N-cadherin retards smooth muscle cell migration and intimal thickening via induction of apoptosis. J Vasc Surg 2010;52:1301-1309.

28. Reddy VS, Valente AJ, Delafontaine $P$, et al. Interleukin-18/WNT1inducible signaling pathway protein-1 signaling mediates human saphenous vein smooth muscle cell proliferation. J Cell Physiol 2011;226:3303-3315.

29. Maekawa T, Komori K, Morisaki K, et al. Ezetimibe reduces intimal hyperplasia in rabbit jugular vein graft. J Vasc Surg 2012;56:1689-1697.

30. Manabe I, Owens GK. The smooth muscle myosin heavy chain gene exhibits smooth muscle subtype-selective modular regulation in vivo. J Biol Chem 2001;276:39076-39087.

31. Weber DS. A novel mechanism of vascular smooth muscle cell regulation by Notch: platelet-derived growth factor receptor-beta expression? Circ Res 2008;102:1448-1450.

32. Doi $H$, Iso $T$, Sato $H$, et al. Jagged1-selective notch signaling induces smooth muscle differentiation via a RBP-Jkappa-dependent pathway. J Biol Chem 2006;281:28555-28564.

33. Dror V, Nguyen V, Walia $\mathrm{P}$, et al. Notch signalling suppresses apoptosis in adult human and mouse pancreatic islet cells. Diabetologia 2007;50:2504-2515.

34. Zhou JX, Han JB, Chen SM, et al. $\gamma$-secretase inhibition combined with cisplatin enhances apoptosis of nasopharyngeal carcinoma cells. Exp Ther Med 2012:3:357-361.

35. Maillard I, Adler SH, Pear WS. Notch and the immune system. Immunity 2003;19:781-791.

36. Stanger BZ, Datar R, Murtaugh LC, et al. Direct regulation of intestinal fate by Notch. Proc Natl Acad Sci USA 2005;102:12443-12448. 